\title{
DEDDWTE

\section{3) Análise de risco de fraturas ósseas em idosas através da Ferramenta FRAX}

\author{
Cristina de Jesus Sousa'; Maria Liz Cunha de Oliveira²
}

\section{Resumo}

Objetivo: Avaliar a qualidade óssea de mulheres com mais de 60 anos por meio da aplicação da Ferramenta FRAX. Método: Trata-se de um estudo descritivo, observacional e transversal com abordagem quantitativa e amostragem de indivíduos idosos, mulheres incluídas de forma consecutiva, desenvolvido em uma clínica ginecológica geral, que presta atendimento a mulheres de todas as regiões do Distrito Federal, na qual foram aplicados um questionário sociodemográfico e a Ferramenta FRAX. Resultados: Observou-se como resultado um risco baixo de fraturas em dez anos em 93,2\%. Já o risco de fraturas médio/alto de 6,8\% foi semelhante a outros estudos. Nas pacientes até 79 anos, o risco de fraturas médio/alto através do FRAX é de 3,7\%, e nas pacientes acima dos 80 anos sobe para 45,5\%. Outra constatação deste trabalho também é quanto ao baixo diagnóstico de osteopenia/osteoporose das pacientes do estudo. Conclusão: Quando se compara as idosas até 79 anos com as idosas acima de 80 anos, existe uma maior variação do percentual de risco de fraturas médio/alto, através do FRAX. Observa-se ainda nas pacientes um alto índice de quedas referidas.

Palavras-chave: Osteoporose. Ferramenta FRAX. Fraturas osteoporóticas. Vitamina D. Idosos.

\section{Introdução}

A osteoporose é uma doença osteometabólica multifatorial que leva à diminuição da massa óssea e da resistência mecânica do osso, aumentando assim o risco de fraturas por fragilidade (PINHEIRO, 2009). Devido à sua característica multifatorial, caráter sindrômico e poucas manifestações clínicas, o diagnóstico de osteoporose torna-se difícil e muitas vezes é feito apenas quando ocorre a fratura (2018).

As fraturas osteoporóticas aumentam substancialmente a mortalidade e a morbidade além de custos elevados aos serviços de saúde e ao governo. É, ainda, a doença osteometabólica mais comum em ambos os sexos, caracterizando-se por baixa densidade óssea e degenera-

1 Mestranda no Programa de Pós-Graduação Stricto Sensu em Gerontologia da Universidade Católica de Brasília, DF, Brasil. Endereço para correspondência: QS 07 Lote 01 - EPCT - 71966-700 - Águas Claras - Taguatinga - DF. Email: cristinadejesussousa@msn.com

2 Professora no Programa de Pós-Graduação Stricto Sensu em Gerontologia da Universidade Católica de Brasília. Email: lizcunhad@gmail.com 
ção da microarquitetura óssea, o que leva a um aumento de fragilidade óssea e, consequentemente, do risco de fratura (AMERICAN MENOPAUSE SOCIETY, 2010).

Embora todos os ossos sejam susceptíveis a fraturas, normalmente elas ocorrem no fêmur, na coluna e no pulso, principalmente na terceira idade. Sendo assim, todo médico que assiste pacientes idosos deve lembrar-se da importância dos fatores associados às fraturas e quedas tais como fratura prévia, sedentarismo, uso de medicamentos que afetam a estabilidade postural e a cognição, desordens neurovegetativas, geometria do quadril alterada, baixa acuidade visual e taquicardia ao repouso (AMERICAN MENOPAUSE SOCIETY, 2010; YAZBEK; MARQUES NETO, 2008).

Segundo as diretrizes brasileiras para o diagnóstico e tratamento da osteoporose em mulheres na pós-menopausa ${ }^{5}$, os fatores de risco mais importantes relacionados à osteoporose e fraturas na pós-menopausa são idade, sexo feminino, etnia branca ou oriental, história prévia e pessoal de fratura, baixa densidade mineral óssea (DMO) no colo do fêmur, baixo índice de massa corporal, uso de glicocorticoide oral, fatores ambientais como o tabagismo e a ingestão acima de 3 unidades/dia de álcool, inatividade física e baixa ingestão dietética de cálcio (RADOMINSKI et al, 2017).

A osteoporose atinge mais de 200 milhões de pessoas no mundo e, segundo a National Osteoporosis Foundation (NOF) (AMERICAN MENOPAUSE SOCIETY, 2010), a cada três segundos ocorre uma fratura osteoporótica, e a cada 22 segundos, uma fratura vertebral osteoporótica.

Para auxiliar em um melhor uso dos recursos para diagnóstico e tratamento da osteoporose, existem várias ferramentas que calculam o risco individual de fratura. Porém, por ser a osteoporose um problema de saúde pública mundial, a OMS solicitou em 2008 à Universidade de Sheffield o desenvolvimento de uma ferramenta, a FRAX (Fracture Risk Assessment Tool) (KANIS, 2008) desenhada para identificar indivíduos que se encontram em alto risco para fraturas por fragilidade óssea, baseando-se em fatores em risco específicos para osteoporose (CENTRE FOR METABOLIC BONE DISEASES, 2017).

Tendo em vista que a osteoporose é um problema de saúde pública que acomete principalmente a população idosa e o seu diagnóstico é realizado pela densitometria óssea, estudos avaliando o risco de fratura devem ser encorajados com o objetivo de melhor a aplicabilidade do FRAX em determinar risco de fratura e início do tratamento específico para osteoporose.

Desta forma o objetivo desta pesquisa é avaliar o risco de fratura osteoporótica por meio da Ferramenta FRAX na população de idosas acompanhadas ambulatorialmente em um serviço de ginecologia.

\section{Métodos}

Trata-se de um estudo descritivo, observacional e transversal com abordagem quantitativa e amostragem incluída de forma consecutiva de indivíduos idosos, mulheres, residentes no DF.

$\mathrm{O}$ estudo foi desenvolvido em uma clínica ginecológica geral, que presta atendimento a mulheres de todas as regiões do DF.

Os critérios de inclusão da amostra foram: mulheres; indivíduos com idade entre 60 e 90 anos, grupo de inclusão da Ferramenta FRAX; e indivíduos que tenham resultado de den- 
sitometria do fêmur realizada há, no máximo 12 meses, exigência da Ferramenta FRAX para ser realizado o cálculo do risco.

Os critérios de exclusão da amostra foram: mulheres incapazes de responder aos questionários; mulheres com prótese bilateral de fêmur ou de joelho.

Para a coleta de dados, foram aplicados dois instrumentos: um questionário sociodemográfico, construído pela pesquisadora e avaliado por três juízes e testado previamente em pacientes que não participaram da pesquisa e a Ferramenta FRAX.

Foram incluídas pacientes com ou sem tratamento prévio de osteoporose.

O presente trabalho foi submetido ao Comitê de Ética em Pesquisa (CEP) da Universidade Católica de Brasília (UCB) e Plataforma Brasil e foi aprovado sob o número CAAE 72085417.8.0000.0029.

\section{Resultados}

Aceitaram participar deste estudo 165 voluntárias, porém preencheram todos critérios apenas 147 voluntárias mulheres, com idade entre 60 e 90 anos. A idade média das idosas é de 67,88 anos. A maioria das voluntárias idosas era moradora de Brasília (70,7\%).

Subdividiram-se, então, as voluntárias em dois grupos; um de qualidade de osso normal e outro com osteopenia/osteoporose obtidos pela densitometria. Foi encontrado baixo risco de fraturas através da aplicação da Ferramenta FRAX em mais de $90 \%$ das pacientes do grupo de osso normal e em 88,5\% das pacientes do grupo de osso osteopenia/osteoporose.

Subdividiu-se o grupo de idosas em dois grupos, o de 60-79 anos e o de $>80$ anos. O número de pacientes acima dos 80 anos correspondeu a 7,5\% das idosas.

Nesse grupo, foram comparadas variáveis pesquisadas nos dois grupos.

Foram observadas mais de $80 \%$ de pacientes autorrelatadas como brancas e sem terapia hormonal.

Foi colocado também o item menopausa e observada sua presença em $98,6 \%$ das pacientes com tempo médio de menopausa de 17 anos nas idosas de 60-79 anos e de 33 anos nas idosas com 80 anos ou mais.

Nesse grupo estudado, viu-se um aumento importante do risco médio ou alto de fraturas ósseas através da aplicação da Ferramenta FRAX, que sai de 3,7\% nas idosas mais novas e vai para 45,5\% nas pacientes com mais de 80 anos, o que denota importante fragilidade das mesmas. No grupo de idosas com menos de 80 anos, foi encontrado densidade mineral óssea normal em 48,5\% das pacientes; nas com mais 80 anos, em apenas $27 \%$. Observou-se, também, um percentual de $34,7 \%$ de pacientes idosas já com história de fraturas prévias.

\section{Discussão}

Tendo como objetivo deste trabalho avaliar a qualidade óssea de mulheres com mais de 60 anos frequentadoras de uma clínica de ginecologia geral no DF, por meio da aplicação da Ferramenta FRAX, observou-se como resultado um risco baixo de fraturas em dez anos em 
93,2\%. Já o risco de fraturas médio/alto de 6,8\% foi semelhante a outros estudos como o resultado encontrado por Bastos-Silva (2016).

Quando se compara as idosas até 79 anos com as idosas acima de 80 anos, existe uma maior variação do percentual de risco de fraturas médio/alto, através do FRAX. Nas pacientes até 79 anos, o risco de fraturas médio/alto através do FRAX é de 3,7\%, e nas pacientes acima dos 80 anos sobe para $45,5 \%$.

Este achado corrobora a posição da American Menopause Society que cita o aumento da taxa de reabsorção óssea logo após a menopausa, indicando uma influência hormonal na densidade óssea das mulheres, levando a uma perda óssea que se acelera dois a três anos antes da última menstruação e que só desacelera três a quatro anos após a menopausa. Assim, por muitos anos, as mulheres perdem $2 \%$ de osso ao ano (AMERICAN MENOPAUSE SOCIETY, 2010).

Looker (2017), em pesquisa desenvolvida nos EUA no período de 2013-2014, em pesquisa com 3127 pacientes sendo 1581 mulheres, com idade média de 57 anos, encontrou FRAX elevado para qualquer fratura em $8 \%$ dos pacientes.

Observa-se nas pacientes um alto índice de quedas nos últimos doze meses, 21,5\%.

Outra constatação deste trabalho diz respeito ao baixo diagnóstico de osteopenia/osteoporose das pacientes do estudo. Enquanto se observa osteopenia/osteoporose em 53,06\% das pacientes idosas, apenas $46,9 \%$ das pacientes têm tratamento prévio para osteopenia/osteoporose, o que mostra oportunidades perdidas de diagnóstico e tratamento de baixa massa óssea, como bem foi alertado por Borges (BACCARO et al, 2013).

$\mathrm{O}$ baixo número de idosos incluídos na pesquisa foi um fator limitante para uma ampla análise pois impossibilitou a divisão de idosos por faixa etária de dez em dez anos como gostaríamos a princípio e nos obrigou a juntar numa mesma coluna FRAX médio e alto para os resultados terem significância estatística.

\section{Conclusão}

É necessária uma abordagem diferenciada à mulher idosa na menopausa quanto à avaliação da qualidade do osso através da densitometria óssea e do risco de fraturas através da Ferramenta FRAX, de fácil aplicabilidade mesmo em nível de saúde básica.

A preocupação com a prevenção de fraturas tem que ser ressaltada pois sabe-se que a taxa de mortalidade no primeiro ano após uma fratura de fêmur é de $10 \%$ a 35\%, e estima-se que apenas 50\% dos indivíduos que sofreram uma fratura de fêmur serão capazes de reestabelecer suas atividades diárias plenamente (YAZBEK; MARQUES NETO, 2008; BACCARO et al, 2013).

Sabe-se também que 20 a $25 \%$ dos pacientes que fraturam o quadril falecem no ano posterior à fratura e outros $25 \%$ nunca mais voltam à sua condição funcional pré- fratura como nos alertam Yasbek e Marques Neto (2008).

Observou-se também um aumento importante do risco de fraturas osteoporóticas com o avançar da idade, o que demonstra fragilidade do grupo de idosas com mais de 80 anos. 


\title{
Risk analysis of bone fractures in the elderly through the FRAX tool
}

\begin{abstract}
Objective: To evaluate the bone quality of women over 60 years of age through the application of the FRAX Tool. Method: This is a descriptive, observational and cross-sectional study with quantitative approach and sampling of elderly individuals, consecutively included women, developed in a general gynecological clinic, which provides care to women from all regions of the Federal District, in which a sociodemographic questionnaire and the FRAX Tool were applied. Results: A low risk of fractures in ten years was observed in $93.2 \%$. The risk of medium / high fractures of $6.8 \%$ was similar to other studies. In patients up to 79 years of age, the risk of medium / high fractures through FRAX is $3.7 \%$, and in patients above 80 years, it rises to $45.5 \%$. Another finding of this work is also about the low osteopenia / osteoporosis diagnosis of the study patients. Conclusion: When comparing the elderly up to 79 years old with the elderly over 80 years, there is a greater variation in the percentage of risk of medium / high fractures, through FRAX. A high rate of falls is still observed in the patients.
\end{abstract}

Keywords: Osteoporosis. FRAX tool. Osteoporotic fractures. Vitamin D. Elderly.

\section{Referências}

AMERICAN MENOPAUSE SOCIETY. Management of osteoporosis in postmenopausal women: 2010 position statement of The North American Menopause Society. Menopause. 2010 jan-fev [acesso em 23 abr 2017];17(1):2324. Disponível em: https://www.ncbi.nlm.nih.gov/pubmed/20061894.

BACCARO, L.F.; MACHADO, V.S.S.; COSTA-PAIVA, L., SOUSA, M.L.; OSIS, M.J.; PINTO-NETO, A.M. Factors associated with fragility fractures in women over 50 years of age: a population-based household survey. Revista Brasileira de Ginecologia e Obstetrícia, Rio de Janeiro, 2013 [acesso em 12 fev. 2017]; 35(11):497-502. Disponível em:http://www.scielo.br/scielo.php?script=sci_arttext\&pid=S010072032013001100004\&lng $=\mathrm{en \& nrm}=\mathrm{iso}$.

BASTOS-SILVA, Y.; AGUIAR, L.B.; PINTO-NETO, A.M.; BACCARO, L.F.; COSTA-PINTO, L. Correlation between osteoporotic fracture risk in Brazilian postmenopausal women calculated using the FRAX with and without the inclusion of bone densitometry data. Archives of osteoporosis, 2016 [acesso em 16 may 2017]; 11(1):1-7.

CENTRE FOR METABOLIC BONE DISEASES. University of Sheffield, 2017 [acesso em: 1ำ mar 2017]. Disponível em: https://www.sheffield.ac.uk/FRAX/.

KANIS, John A. Frax. Instrumento de avaliação do risco de fratura, 2008. [acesso em 9 ago 2016]. Centre for Metabolic Bone Diseases, University of Sheffield, UK. Disponível em: https://www.shef.ac.uk/FRAX/tool.js$\mathrm{p}$ ?lang $=\mathrm{pt}$

LOOKER, A.C.; ISFAHANI, N.S. FRAX-based Estimates of 10-year. Probability of Hip and Major Osteoporotic Fracture Among Adults Aged 40 and Over: United States, 2013 and 2014. National Health Statistics Reports, 2017 [ acesso em 1ํo abr 2017]; 103:1-16. Disponível em: https://www.ncbi.nlm.nih.gov/pubmed/28459415.

PINHEIRO, M.M.; CAMARGOS B.M.; Borba V.Z.C.; LAZARETTI-CASTRO, M. FRAX TM: construindo uma ideia para o Brasil. Arq Bras Endocrinol Metab [internet]. 2009 ago [acesso em 12 fev 2017];53(6):783-790. Disponível em: http://www.scielo.br/scielo.php?script=sci_arttext\&pid=S000427302009000600015\&lng=en\&nrm =iso.

RADOMINSKI, S.C.; BERNARDO, W.; PAULA, A.P.; ALBEGARIA, B.H.; MOREIRA, C.; FERNANDES, C.E. et al. Diretrizes Brasileiras para o diagnóstico e tratamento da osteoporose em mulheres na pós-menopausa. Revista Brasileira de Reumatologia. 2017 [acesso em 25 mar 2018);57(2):452-466. Disponível em: https://doi. org/10.1016/j.rbr.2017.06.001

SOUZA, M.P.G. Diagnóstico e tratamento da osteoporose. Rev Bras de Ortopedia, 2010 [acesso em 30 abr. 2018]; 45(3):220-229. Disponível em: https://doi-org.ez97.periodicos.capes.gov.br/10.1590/S0102-36162010000300002. 
YAZBEK, M.A.; MARQUES NETO, J.F. Osteoporose e outras doenças osteometabólicas no idoso. Einstein. 2008 [acesso em 28 abr 2018];6(1):S74-S78. Disponível em: http://bases.bireme.br/cgi-bin/wxislind.exe/iah/online/?IsisScript $=$ iah/iah $. x i s \& s r c=$ google\&base $=$ LILACS\&lang $=$ p\&nextAction $=\operatorname{lnk} \&$ exprSearch $=516983 \&$ indexSear $\mathrm{ch}=\mathrm{ID}$. 